\title{
Diagnostic Limitations of Cephalometrics in Orthodontics-A Review
}

\author{
Dr. Appasaheb Naragond ${ }^{1}$, Dr. Smitha Kenganal ${ }^{2}$, Dr. Roshan Sagarkar ${ }^{3}$, \\ Dr. N. Sathish Kumar ${ }^{4}$, Dr.Sugaradday ${ }^{5}$ \\ ${ }^{I}$ (Department of Orthodontics \&Dentofacial Orthopedics P.M.N.M Dental College \& Hospital Bagalkot, Rajiv \\ Gandhi university of health sciences Karnataka India) \\ ${ }_{2}^{2}$ (Department of Conservative Dentistry \& Endodontics P.M.N.M Dental College \& Hospital Bagalkot, , \\ Rajiv Gandhi University of health sciences Karnataka India) \\ ${ }^{3}$ (Department of Orthodontics \& Dentofacial Orthopedics Ramayya Dental College \& Hospital Bangalore, \\ Rajiv Gandhi University of health sciences Karnataka India) \\ ${ }^{4}$ (Sri Manakula Vinayagar Medical College and Hospital Kalitheerthalkuppam, Puducherry.Tamil Nadu \\ India) \\ ${ }^{5}$ (Department of Orthodontics \& Dentofacial Orthopedics Navodaya Dental College, Raichur Rajiv Gandhi \\ university of health sciences Karnataka India)
}

\begin{abstract}
Since from the introduction of cephalometric analysis in diagnosis and orthodontic treatment planninjg, it plays a major role. So many efforts have been made by various studies to standardize the values for different reces, to minimize the distortion and magnification, to create universally accepted analysis, even after this still so many limitations are there in diagnosis of orthodontic treatment. So in this review we have made effort to enlight such limitation and aware during planning treatment using cephalometric analysis.
\end{abstract}

\section{Introduction}

Since we as orthodontist are after all in health service, our first consideration should be the welfare of the patient. This welfare of the patient is determined by whether the services rendered to the patient were constructive and did the improvement warrant the orthodontic procedure involved. The result of the orthodontic therapy- Good, Bad, or indifferent depends upon the limitations in our diagnosis, treatment planning and mechanics. These limitations may either result in success or failure of orthodontic treatment.

Treatment goals that are well defined are an absolute necessity for us to proceed in the right direction. We are already in the 21 st Century and it is time for us to move to the next level to the demands of our patients. We need to more clearly define out treatment goals and eliminate some of the aids that we have in trying to decide which treatment plan and which treatment approach we are going to use on our patient . To quote Yogi Berra "If you don't know where you are going you will probably end up somewhere else"

Since the introduction of cephalometrics radiography in 1931, considerable progress has been achieved over the years in standardizing equipments and techniques. This is to minimize the effects of distortion and magnification i.e. the errors of projection. In cephalometrics there are different sources of errors. These errors must be quantified in order to substantiate the validity of cephalometric research and clinical applications. Houston $^{1}$ (1983) classified the cephalometric error into systematic error and random error. The systematic error is a result of the two dimensional representation of a three-dimensional object, it is subjected to distortion. Since the rays are not parallel and diverging from a small source onto the film, it is subjected to enlargement (Houston, 1983). ${ }^{1}$ The magnitude of enlargement depends on the relative distance between the film and the $\mathrm{x}$ ray source. The further the film is from the object, the greater the enlargement (Bregersen, 1980). ${ }^{2}$ The random error can arise as a result of variations in positioning of the patient's head in the cephalostat. The soft tissue points in particular are affected by the way the patient is positioned; variations in the film density and sharpness also lead to random errors ${ }^{1}$ (Housten, 1983).

\subsection{General Limitations Of Cephalometrics}

1. It gives a two dimensional view of a three dimensional object. ${ }^{3,4}$

2. There can be errors in identification of landmarks. Thus reliability of cephalometrics comes down.

3. Errors can be made during tracing procedures.

4. Assumptions: Various things are "assumed" in cephalometrics.

a) Symmetry: The various analysis done on lateral projections are based on the assumptions that the patient does not have any skeletal asymmetry then the results of the analysis may not be accurate. This can be avoided by analysis of postero-anterior projections. 
b) A correct occlusal and postural position is important in the accuracy of the cephalogram.

5. Fallacy of false precision It is found that when a person takes a series of cephalogram of the head of the same person and does the tracing, locates the land marks and calculates ; various angles measured showed a standard error of 1:5 that is each time it is slightly different.

6. Fallacy of ignoring the patient: The cephalometric values should not be taken as fixed goals. sometimes certain values of a given patient may vary from the mean value. But it may not be an indication for treatment. Thus the patient should be analyzed individually before a treatment plan is laid. Just because the values differ it doesn't mean that treatment is required. If functions and esthetics are satisfied any deviations from normal of the cephalometrics value can be ignored.

\subsection{Critical Limitations Of Cephalometrics -}

Down's,5 in 1948 was the first to suggest that skeletal and dental picture could be analyzed by comparing tracings, since then a considerable number of such analysis have been developed. Lateral skull radiographs are a very useful tool in the completion of diagnosis, but they are only an aid to be taken with other radiographs, study models and most important the clinical examination of the patient.Direct linear measurements are not popular, since head vary in size and some enlargement is inevitable in producing the radiographs. It is necessary to have data with which to compare the figures measured in the individual under consideration. However, the measurements made by the tracings are by no means exact, studies have shown considerable variations and " error of method " as assessed by a number of investigations.

Studies using digitizer were found to be more accurate than the conventional method. The error of method is a statistical deviation similar to a standard deviation. Thus if the error method for angle SNA is $0.60^{\circ}$ this means that approximately two-third of individuals one's tracings will be within $0.60^{\circ}$ of the correct value. In $95 \%$ of individuals it will be within a usage of twice this figure and in virtuality all the individuals it will be within a range of three times this figure. Because of the wide range found in any study there is no point in giving a standard value to two places of decimals. Various investigations have reported samples of varying sizes and ages, in some cases the samples were selected while in other cases it was random sample selection.

\subsection{Individual Limitations}

\section{Down's analysis -}

Down's,5 recognized that racial profile could be retrusive or protrusive yet be harmonious in proportions. This led him to formulate his analysis. Down's obtained his normal values of ranges from a very small sample of just 20 subjects compromising of only Caucasian subjects. Hence a wide variation can be expected between different races and people. Since the study was restricted to the age group of 12 to 17 years, it may not be very well applicable to the adults, as age changes can be also be expected.

Studies conducted by Sheldon Baurind ${ }^{6}$ in 1971, using "Scattergrams" showed that considerable variations occur in the locations of the points forming the Frankfort Horizontal plane i.e. the porion and orbitale. Besides this the other points used can also vary considerably, In the facial angle which gives the degrees of retrusion or protrusion of the lower jaw, certain errors of interpretations should be avoided in cases having unusual locations such as the nasion in small orbitale cavities or when anomalies lie in the temporal bone. One frequently expressed problem was the use of the machine ear post porion, therefore it is always advisable to use the true ear hole and locate a point at the top of the external auditory meatus. Because in a study by Bjork, Backland and Perrera to find out the error of method at source it was found that the error from the source are negligible except for in case of the porion. ${ }^{7}$

The facial convexity or the angle of convexity can also be subjected to limitations and hence Rickett's ${ }^{8}$ suggest that it would be advisable to measure the distance of point A directly into a straight line drawn from the N-Pog. Similarly Y-axis which encompasses the sella can also vary due to identification of the mid point of Sella-Tursica, which is nothing more than a housing for the hypophysis and the pituitary gland in the brain and is in no other way related to the jaws. In the same light Ricketts states the preferability to the use of frankfort horizontal plane rather than the $\mathrm{S}-\mathrm{N}$ plane. The location of the occlusal plane can also be subjected to variations, because when considering the denture pattern, the occlusal plane bisects the overlapping cusp of the 1st molars and the incisal overbite hence, grossly malpositioned teeth can create difficulties in locating this plane. Similarly in cases of crowding the location of the incisal tip can be subjected to inaccuracies and do not identify individual teeth.

Downs timely warning. It was he who had shown that discrepancies between cephalometric facial typing and photographic facial typing disappear when the Frankfort plane is not horizontal but tilted up or down. 


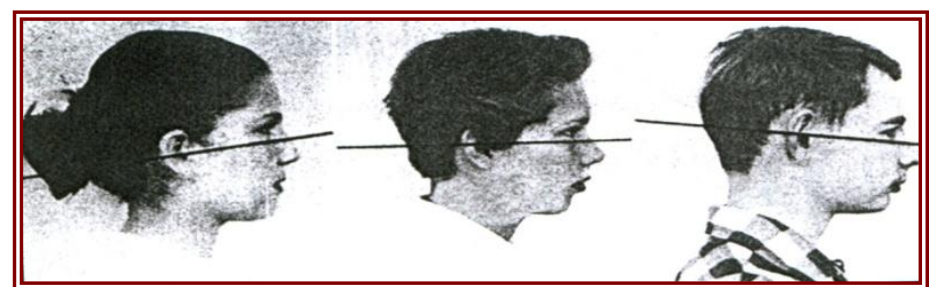

2) Steiners analysis ${ }^{9-11}$ - The reference plane here is the $\mathrm{S}-\mathrm{N}$ plane which can be subjected to discrepancies due to the points constructing the plane. The sella and the nasion are both situated in the interiors of the skull and cannot be visualized clinically and therefore cannot be utilized as a tool for actual direct clinical communications. This plane when compared to the frankfort horizontal plane which has both the porion and orbitale are located in the external position of the skull, and follows the soft tissue orbit and the ear post respectively both of which can be visualized clinically. Although nasion bears anatomic significance to the face, se11a in contrast boars none. As the housing for the hypophysis of the brain and the pituitary gland, sella is totally unrelated and not relevant to the face or the jaws in particular. The sella-nasion does not serve as a basic framework of the anterior cranial base and fossa which is the super structure scaffolding of the nasal capsule or the face. However, it bears little significance to the mandible and its morphology. The mid-point of the sella tursica is determined in space and hence Hunter likes to call it a "space mark" instead of a "land mark" as it lacks a craniometric definition.

The morphology and orientation of the superior border of the dorsum sella, including both the posterior clinoidal processes were studied in 21 cases and it was found that the superior border of the dorsum sella formed a plane, In 16 of the cases the posterior clinoidal processes were superior to the median structures and in 11 of the cases they were more inferior. Contour gauges were used to study the posterior and the inferior surfaces of the hypophyseal fossa, the tuberculum sella, and the relationship of the latter to the anterior clinoidal processes. This showed a relatively large variation in transverse width from 12 to $22 \mathrm{~mm}$. The image on the cephalogram was frequently somewhat obscured by the projection of the anterior clinoidal processes. The image of the tuberculum Sella and the anterior structure representing the plenum sphenoidale is one of the most clearly defined structure in the skull-. The midsagital contour of the hypophyseal fossa and its surrounding structure in general can be detected accurately on the lateral cephalogram. The posterior internal wall on the superior border of the dorsum sella, frequently divides into 2 or 3 lines out of which the most dorsal and the inferior one have to be given preference.

Considering the nasion point, in many cases it may deviate from its mid sagital plane due to asymmetry of the nasal bones. Usually variations cannot be detected in the radiographs because it results in relative broadening of the image at the nasion point thus loosing its clearance. When the image of the anterior part of the suture is not distinguishable, Hunter suggest taking the most posterior point on the curve as the nasion The SNA and SNB angles play fundamental roles in a number of cephalometric analysis. As far as the mean values of the SNA angles are concerned, the male and female samples agree quite closely but the variance are consistently significantly greater for females. The significance of angle ANB varies according to the size of the angle SNA, it has been suggested that for every one degree that SNA falls below the standard value half a degree should be substracted from the ANB of $3^{\circ}$ and half a degree should be added to every degree of increase. However, this correction is not fool proof

The point A varies invariably due to the outline of the alveolar bone extending from the central incisor composing the inferior aspect which is usually not very clearly visible in the radiograph. The anterior nasal spine plays an unjustifiying role in the determination of point $\mathrm{A}$, as it can affect the vertical and anteroposterior location of point $\mathrm{A}$ and its variation reflect heavily on the location of point $\mathrm{A}$.The point $\mathrm{B}$, also varies considerably in its vertical relationship to the apex of the central incisor. In most cases determination in relation to the mandibular border places the point more in the region of the apices. 


\section{Shortcomings of ANB angle:}

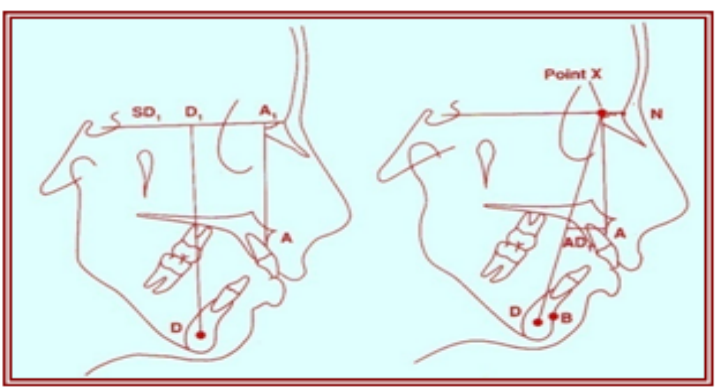

Taylor ${ }^{12}$ in 1969 pointed out that ANB angle did not always indicate true apical base relationship. Varied horizontal discrepancies of points $A$ and $B$ could give the same ANB measurement because variation in the vertical distance from nasion could compensate for other variation.Beatty ${ }^{13}$ in 1975 reported that ANB angle is not always an accurate method of establishing the actual amount of apical base divergence. As an alternative to ANB angle for measuring apical base discrepancy, he devised the $\mathrm{AXD}$ angle, where point $\mathrm{x}$ is located by projecting point A on to a perpendicular to SN line. Point $\mathrm{D}$ is located in the bony symphysis as described by Steiner

\section{Wits appraisal ${ }^{14}$}

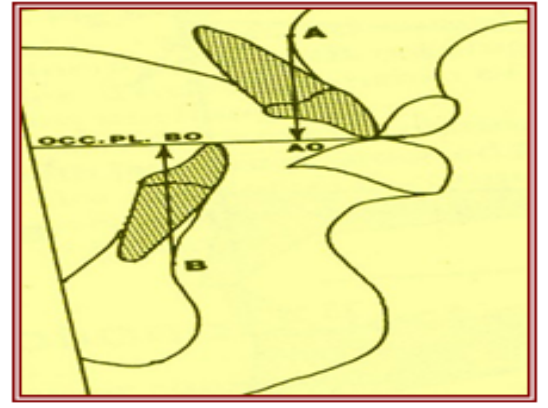

This analysis takes into consideration the occlusal plane to determine the anteroposterior relationship of the jaws. This plane can vary with malpositioning of the teeth, besides this it is not a diagnostic aid and is only useful to understand the A-P jaw relationship when the angle ANB doesn't provide a very clear image and in limitation that the mandibular plane is between $27^{\circ}$ to $37^{\circ}$

Rakosi's Analysis $^{15}$ - This analysis for functional therapy is undertaken when growth potential is still present and can be subject to considerable variations and changes.

The point used for the saddle angle i.e. N, S, Ar are all subject to variations. The sella also undergoes considerable changes during growth. The landmarks used for articular angle and gonial angle are also variable, though they indicate the position of the mandible and give the growth direction, it has to be considered in conjunctions with other findings, since sudden changes in the pattern of growth are not uncommon. Since all the measurements are made taking mandible into and around the interpositioned dentition, which has a very important role to play especially with the mixed dentition and eruption not yet complete, considerable differences may be brought about by the occluding dentition as stated by Van der Linden, who states the importance of the developing dentition in affecting various malocclusions. The other major drawback being that the growth is assessed in a two dimensional direction i.e. anteroposteriorly thus leaving other vital aspects of growth, i.e in the transverse direction.

McNamara Analysis ${ }^{16}$ - McNamara based his studies on 3 previous studies by Bolton, Burlington and Ann Arbour; all of which represent considerable variations between them. Those studies were restricted to specific populations and may not be applicable in every sense to different populations.

Bjork's Analysis $^{7}$ - Bjork based his study on the Swedish population and like Rakosi's analysis makes use of many parameters which may vary for different populations. Bjorks studies of facial prognathism also illustrates the unreliability of intra cranial reference lines on cephalograms. Two adult Bantu men were selected to represent maximum and minimum facial prognathism relative to the S-N plane. Bjork illustrates the greatest variation in the inclination of the cranial base rather than the greatest differences in prognathism.

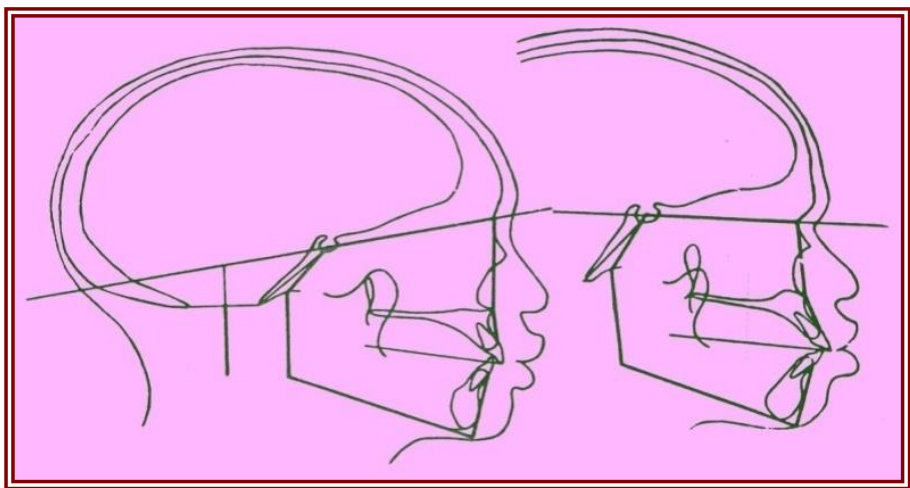

www.iosrjournals.org 
Wylie's Analysis ${ }^{17}$ - Gives an anteroposterior dysplasia in. horizontal plane. All measurements are made parallel to the Frankfort horizontal plane, which as discussed earlier is subject to considerable changes.

Holdaway's Analysis $^{18}$ - Takes into consideration soft tissue balance and harmony. As with any other soft tissue landmarks, identification is not very easy. The tonicity and muscular tensions cannot be assessed easily.

The Margoli 's maxillofacial triangle ${ }^{19}$ - This triangle makes it possible to determine growth and overall facial growth patterns. But any changes in a single point can alter drastically the outcome, Since it depends on a triangular pattern i.e. a shift in a point such as $\mathrm{M}$ can lead to a considerable variation.

The Profile Analysis of Shwarz ${ }^{15}$ - This analysis depends upon the N-perpendicular and the FH, both of which are highly variable and also certain soft tissue landmarks such as the subnasale may not be clearly identifiable.

Sassouni's Analysis. ${ }^{20}$ - This analysis makes use of the arcs and the planes and states that these arcs and planes indicate deep bite or open bite, but this may not be true because the dental and skeletal components have not been considered separately; whereby even though the palatal plane and the mandibular plane may be divergent but the difference may be dentally compensated examples like this have not been taken into account in this analysis.

The Morrees Mesh Diagram ${ }^{21}$ - Here the reference landmarks such as the Nasion and the Sella which are considered are highly unstable, besides this establishing the true vertical is very important in this analysis. Any error can lead to errors in interpretations and it is more suitable for individual longitudinal studies only. Also when the cranial base has moved upwards and downwards, the SN plane cannot be considered for this analysis.

The Quadrilateral Analysis ${ }^{22}$ - This study of De Paulo was based on adolescent patient and confines itself primarily with the skeletal configuration. Difficulty and variations can arise in locating point "J".

Cephalometrics for Orthognathic Surgery ${ }^{23}$ - This analysis given by Burstone may confine itself to limited populations and the norms given in this study may vary in different populations. The main reference plane drawn at $7^{\circ}$ to $\mathrm{SN}$ plane can be subjected to changes as in the $\mathrm{S}$ and $\mathrm{N}$ positions The cranial base is measured from Ar and any adversity in the position of the mandible could lead to an elevated reading. It also fails to take into consideration the transverse relationships and discrepancies.

\section{Conclusion}

In conclusion, it can be stated that the orthodontist should have more conservatism to his treatment approach. Because like all practioners in specialized areas, even orthodontists tend to over treat their patients, and develop a guilt at the end of the treatment. This guilt is not because of mercenary motives, but guilty because of eternal optimism, that makes the orthodontist feel that he/she will be able to do more than we actually accomplish in the end. Hence, we must look more realistically and objectively at our shortcomings and our failures, and devote more of our research energies in an attempt to supply the answers to some of these basic problems that plague the clinical orthodontist. "

On account of glitter of the new developments and the rhetoric of the proponents developing them, we are likely to turn a blind eye to the previous treatment modalities, concepts are hardware components. It would be a folly to do so .No new development can be perfect, and no older approaches are totally useless . If we keep our eyes and mind open to integrate to the good aspects of all systems judiciously in such way that the biomechanical principles are not violated, the combinations can over come serious draw back of any appliance we wish to use in our practice. This would make the treatment more efficient and reduce the biologic risks

\section{References}

[1] Houston W. The analysis of errors in orthodontic measurements. Am J Orthod1983;83:382-90.

[2] Bergersen EO. Enlargement and distortion in cephalometric radiography. Compensation tables for linear measurements. Angle Orthod 1980; 50:230-44.85

[3] Reiten Karre. As citied in "Biochemical principal and reaction" Graber and Swain, Mosby,1991

[4] Bholaji S.I. As citied in "Cephalomateic file"

[5] Graber T.M. In "Clinical alterations of growing face". Monoorgraph no. 12

[6] Baumrind S, Frantz R. The reliability of head film measurements. 1. Landmark identification. Am J Orthod 1971;60:111-27. 
[7] Björk A. The relationship of the jaws to the cranium. In: Lundstrom A, ed. Introduction to orthodontics. New York: McGr aw Hill Book Company, 1960:104-40.

[8] Ricketts R. Perspectives in the clinical application of cephalometric. Angle Orthod 1981;51:115-50.

[9] Steiner CC. Cephalometrics for you and me. Am J Orthod 1953;39:729-55.

[10] Steiner CC. Cephalometrics in clinical practice. Angle Orthod 1959;29:8-29.

[11] Steiner CC. The use of cephalometrics as an aid to planning and assessing orthodontic treatment. Am J Orthod 1960;46:721-35.

[12] Taylor, C.M., 1969. Changes in the relationship of nasion, point A and point B and the effect upon ANB. Am J Orthod., 56: 143-63

[13] Beatty, E.J., 1975. A modified technique for evaluating apical base relationships. Am J Orthod., 68: 303-15.

[14] Jacobson A. The wits appraisal of jaw disharmony. Am J Orthod 1975;67:125-38.

[15] Thomus Rakosi-An atlas and manual of cephalometric radiography 1982

[16] McNamara J. A method of cephalometric analysis. In clinical alteration of the growing face, monograph 12, craniof acial growth series. Ann Arbor, 1983, University of Michigan, Centre for Human Growth and Development.

[17] Wylie.LWindell.The assessement ofanterioposterir dysplasia.Angle Ortho 1947 17-109

[18] Holdaway RA. Changes in relationship of point A and point B during orthodontic treatment. Am J Orthod. 1956; 42: 176-93.

[19] Margol H I A basic facial pattern and its appliacation in clinical orthodontics; I maxilla facial triangle J Orthodand Oral Surgery, Vol.33, No,10 Oct;pp631-641

[20] Sassouni V. A classification of skeletal facial types. Am J Orthod 1969;55:109-23.

[21] Moorrees C, Lebret L. The mesh diagram and cephalometric. Angle orthod 1962;32:214-31

[22] De.paulo et al.The Quadrilateral Analysis, An individualizedskeletal assessment Am J orthod S.Louis Jan 1983.V 83, N.1P.19-32

[23] Burstone, C.J., James, R.B., Legan, H., Murphy, G.A., Norton, L.A., Farmington, C., 1978. Cephalometric for orthognathic surgery. J. Oral Surg. 36, 269-277. 